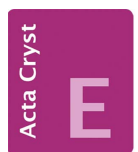

CRYSTALLOGRAPHIC COMMUNICATIONS

ISSN 2056-9890

Received 30 September 2016

Accepted 25 October 2016

Edited by R. F. Baggio, Comisión Nacional de Energía Atómica, Argentina

Keywords: crystal structure; pharmaceutical compound; 5-aminopyrazoles; nucleophilic substitution; hydrogen bonding.

CCDC reference: 1511522

Supporting information: this article has supporting information at journals.iucr.org/e

\section{Crystal structure of 5-butylamino-3-methyl-1- (pyridin-2-yl)-1H-pyrazole-4-carbaldehyde obtained from a microwave-assisted reaction using caesium carbonate as catalyst}

\author{
Mario A. Macías, ${ }^{a}$ Jessica Orrego-Hernández ${ }^{\mathbf{b}}$ and Jaime Portilla ${ }^{b_{*}}$
}

${ }^{\mathbf{a}}$ Department of Chemistry, Universidad de los Andes, Carrera 1 No. 18A 10, Bogotá, Colombia, and ${ }^{\mathbf{b}}$ Bioorganic Compounds Research Group, Department of Chemistry, Universidad de los Andes, Carrera 1 No. 18A 10, Bogotá, Colombia. *Correspondence e-mail: jportill@uniandes.edu.co

The title compound, $\mathrm{C}_{14} \mathrm{H}_{18} \mathrm{~N}_{4} \mathrm{O}$, synthesized from an unconventional microwave-assisted method using caesium carbonate as catalyst, has an approximately planar conformation with the pyridyl and pyrazole rings inclined by a dihedral angle of $7.94(3)^{\circ}$, allowing the formation of an intramolecular $\mathrm{N}-\mathrm{H} \cdots \mathrm{N}$ hydrogen bond. The supramolecular assembly has a three-dimensional arrangement controlled mainly by weak $\mathrm{C}-\mathrm{H} \cdots \mathrm{O}$ and $\mathrm{C}-\mathrm{H} \cdots \pi$ interactions.

\section{Chemical context}

Pyrazole derivatives are compounds with notable biological activity (Peng et al., 2013) and some derivatives have the capacity to form complexes with metal ions (Budzisz et al., 2009). Currently, 5-aminopyrazoles have been found to play an important role as biologically active compounds (Zhang et $a l ., 2014)$. As such, they are considered to be building blocks of high interest for pharmaceutical agents (Sakya et al., 2006) and agrochemicals (Yuan et al., 2013). Recently, our research group reported the chemoselective synthesis of 5-alkylamino$1 H$-pyrazole-4-carbaldehydes in which $\mathrm{C}-\mathrm{N}$ bond formation in pyrazole rings were efficiently assisted by using caesium carbonate under microwave irradiation with short reaction times and excellent yields (Orrego-Hernández et al., 2015a). Herein, we report the crystal structure of the new 5-(butylamino)-3-methyl-1-(pyridin-2-yl)- $1 H$-pyrazole-4-carbaldehyde derived from 5-chloro-3-methyl-1-(pyridin-2-yl)- $1 H$ pyrazole-4-carbaldehyde and butylamine by using the 'caesium effect' and microwave irradiation.

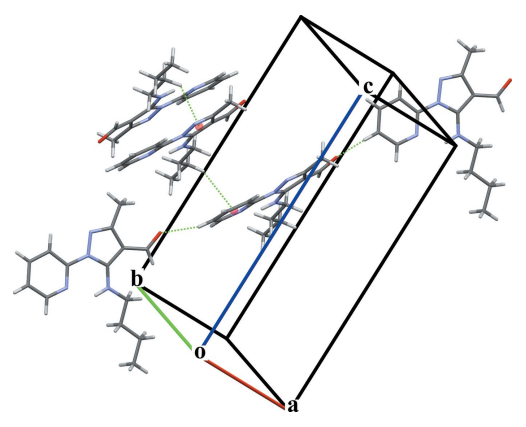

OPEN $\odot A C C E S S$

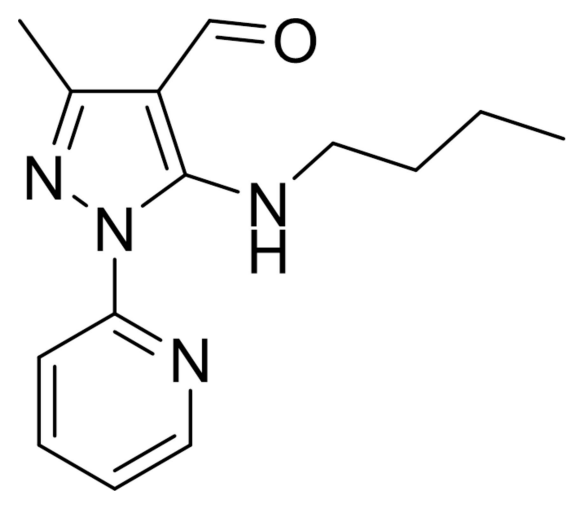




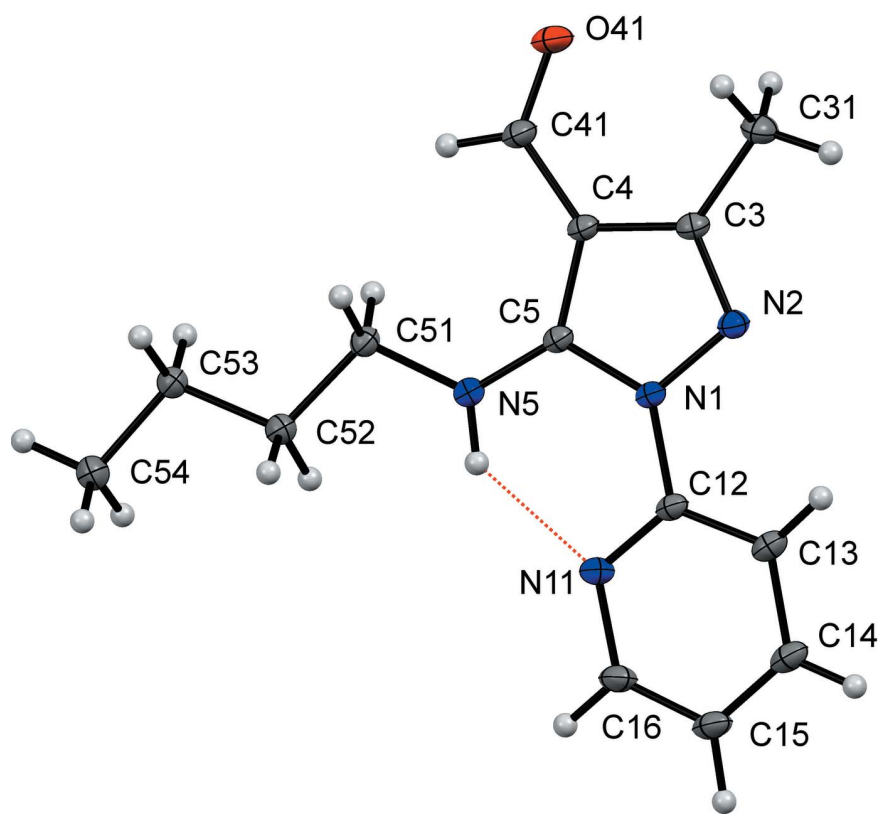

Figure 1

The molecular structure of the title compound, showing anisotropic displacement ellipsoids drawn at the $50 \%$ probability level. The intramolecular $\mathrm{N}-\mathrm{H} \cdots \mathrm{N}$ hydrogen bond is shown as a dashed line (see Table 1).

\section{Structural commentary}

In the molecular structure of the title compound (Fig. 1), the pyridyl and pyrazole rings are nearly coplanar with a dihedral angle between their planes of $7.94(3)^{\circ}$. The pyridyl ring has an orientation that allows the formation of an intramolecular N5-H1 $\cdots$ N11 hydrogen bond (Fig. 1 and Table 1) to generate an $S(6)$ motif. This structural feature is also observed in its analog 5-cyclohexylamino-3-methyl-1-(pyridin-2-yl)- $1 \mathrm{H}$ pyrazole-4-carbaldehyde, which even shows a smaller dihedral angle between the pyridyl and pyrazole rings [2.47(5)

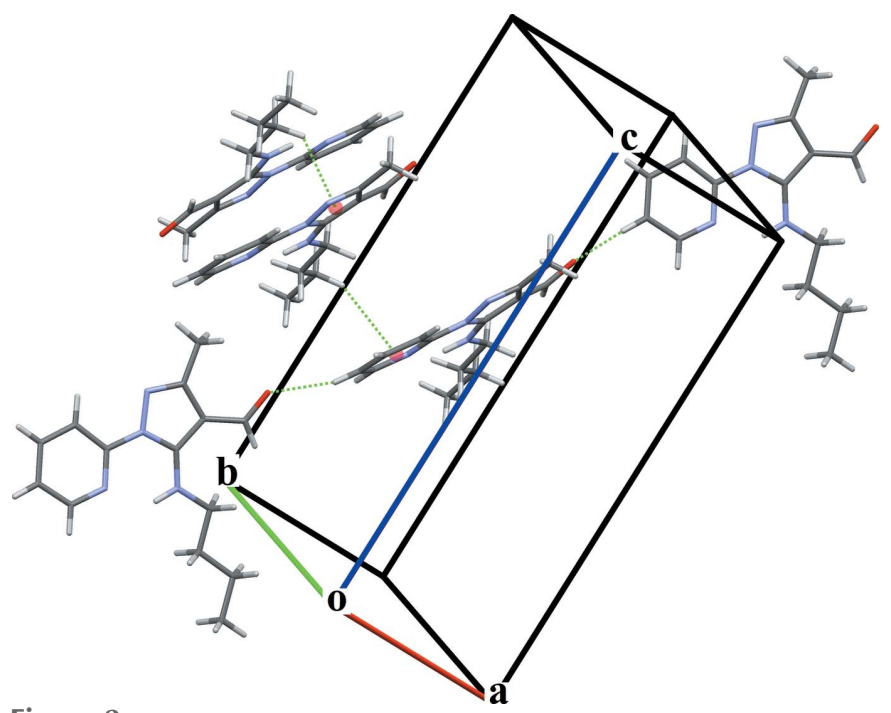

Figure 2

The crystal structure of the title compound, showing the $\mathrm{C}-\mathrm{H} \cdots \mathrm{O}$ and $\mathrm{C}-\mathrm{H} \cdots \pi$ hydrogen-bond interactions.
Table 1

Hydrogen-bond geometry $\left(\AA,^{\circ}\right)$.

$C g 1$ abd $C g 2$ are the centroids of the $\mathrm{C} 3-\mathrm{C} 5 / \mathrm{N} 1 / \mathrm{N} 2$ and $\mathrm{N} 11 / \mathrm{C} 12-\mathrm{C} 16$ rings, respectively.

\begin{tabular}{lllll}
\hline$D-\mathrm{H} \cdots A$ & $D-\mathrm{H}$ & $\mathrm{H} \cdots A$ & $D \cdots A$ & $D-\mathrm{H} \cdots A$ \\
\hline $\mathrm{N} 5-\mathrm{H} 1 \cdots \mathrm{N} 11$ & $0.88(1)$ & $2.00(1)$ & $2.7117(7)$ & $137(1)$ \\
$\mathrm{C} 15-\mathrm{H} 15 \cdots \mathrm{O} 41^{\mathrm{i}}$ & 0.95 & 2.36 & $3.2906(8)$ & 165 \\
$\mathrm{C} 52-\mathrm{H} 52 B \cdots C g 1^{\mathrm{ii}}$ & 0.99 & 2.77 & $3.5141(6)$ & 132 \\
$\mathrm{C} 53-\mathrm{H} 53 A \cdots C g 2^{\mathrm{iii}}$ & 0.99 & 2.98 & $3.8761(6)$ & 152 \\
\hline
\end{tabular}

Symmetry codes: (i) $x-1,-y+\frac{3}{2}, z-\frac{1}{2}$; (ii) $-x+1,-y+1,-z+2$; (iii) $x+1, y, z$.

Orrego-Hernández et al., 2015b). In both molecules, the 3methyl-1-(pyridin-2-yl)-1 $H$-pyrazole-4-carbaldehyde nucleus presents a similar, but not identical, conformation with a maximum r.m.s. deviation of $0.0906 \AA$, keeping the atomic distances very similar in the pyrazole ring.

\section{Supramolecular features}

In the crystal structure, $\mathrm{C} 15-\mathrm{H} 15 \cdots \mathrm{O} 41^{\mathrm{i}}$ [symmetry code: (i) $\left.x-1,-y+\frac{3}{2}, z-\frac{1}{2}\right]$ interactions link the molecules into $C(10)$ chains running along [201], see Fig. 2. Parallel chains are connected by weak $\mathrm{C} 52-\mathrm{H} 52 \mathrm{~B} \cdots \mathrm{Cg} 1^{\mathrm{ii}}[\mathrm{Cg} 1$ is the centroid of the $\mathrm{C} 3-\mathrm{C} 5 / \mathrm{N} 1 / \mathrm{N} 2$ ring; symmetry code: (ii) $-x+1,-y+1$, $-z+2]$ and $\mathrm{C} 53-\mathrm{H} 53 A \cdots C g 2^{\mathrm{iii}}[\mathrm{Cg} 2$ is the centroid of the $\mathrm{N} 11 / \mathrm{C} 12-\mathrm{C} 16$ ring; symmetry code: (iii) $x+1, y, z]$ interactions, which help to define a three-dimensional array.

\section{Database survey}

A search of the Cambridge Structural Database (CSD Version 5.37 with two updates; Groom et al., 2016) for the 1-(pyridin-2yl)- $1 H$-pyrazole nucleus with the possibility of any group bonded to $\mathrm{C} 3, \mathrm{C} 4$ or $\mathrm{C} 5$ gave 12 hits of which 10 correspond to organometallic compounds, one to 2-(3,5-bis(4-(n-octyloxy)phenyl)pyrazol-1-yl)pyridine and the last to 2,6-bis(pyrazol(pyrazolyl)pyridine. Any other search considering the presence of the butylamino or carbaldehyde groups gave no hits. However, two related compounds 5-cyclohexylamino3-methyl-1-(pyridin-2-yl)- $1 H$-pyrazole-4-carbaldehyde and (Z)-4-[(cyclohexylamino)methylidene]-3-methyl-1-phenyl$1 H$-pyrazol-5(4H)-one have been published recently (OrregoHernández et al., 2015b). These compounds are pyrazole derivatives which, despite the overall similarities of the molecular geometries and the potentially available donors and acceptors for hydrogen-bonding interactions, present different supramolecular assemblies.

\section{Synthesis and crystallization}

All reactive and solvents, including caesium carbonate (99\%, Aldrich), were purchased from commercial sources and used as received. A mixture of 5-chloro-3-methyl-1-(pyridin-2-yl)$1 H$-pyrazole-4-carbaldehyde [(I) in Fig. 3; 0.100 g, 0.45 mmol, 1 equiv.], butylamine [(II) in Fig. 3; $0.56 \mathrm{mmol}, 1.3$ equiv.], caesium carbonate $(0.029 \mathrm{~g}, 20 \% \mathrm{mmol}, 0.2$ equiv. $)$ and $2 \mathrm{~mL}$ 


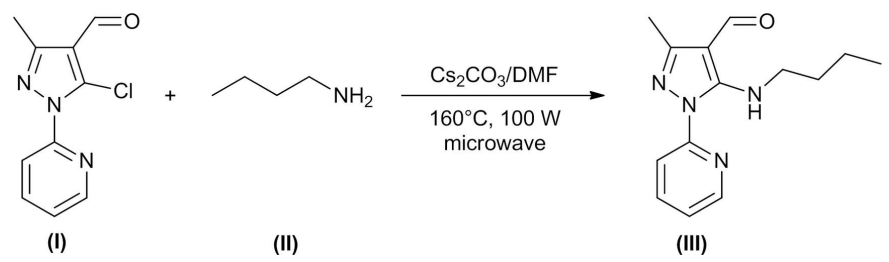

Figure 3

Schematic representation of the microwave-assisted reaction using caesium carbonate as catalyst.

of dimethylformamide (DMF) were placed in a reaction tube of a CEM DiscoverTM, containing a magnetic stirring bar. The tube was sealed with a plastic microwave septum and was irradiated at $433 \mathrm{~K}$ for $25 \mathrm{~min}$ at $100 \mathrm{~W}$. The resulting crude product was partitioned between dichloromethane and water. The organic layer was washed with water, then brine, and dried over anhydrous sodium sulfate. Subsequently, the solvent was removed under vacuum and the residue was purified by silica gel flash chromatography (DCM) to afford 5-(butylamino)-3-methyl-1-(pyridin-2-yl)-1 $H$-pyrazole-4-carbaldehyde [(III) in Fig. 3]. Yellow crystals of (III) suitable for single-crystal X-ray diffraction were grown in DMF by slow evaporation, at ambient temperature and in air, [94\% yield, m.p. $354 \mathrm{~K}]$. HRMS (ESI+): $[M+\mathrm{H}]^{+}$calculated for $\mathrm{C}_{14} \mathrm{H}_{19} \mathrm{~N}_{4} \mathrm{O}^{+}$259.1553, found 259.1546. Yield $0.109 \mathrm{~g}, 94 \%$; m.p. 348-350 K; IR $v_{\max }(\mathrm{KBr}): 3448,3211,3096,2924,2858$, $1643,1596,1563,1436,1002 \mathrm{~cm}^{-1} ;{ }^{1} \mathrm{H}$ NMR $\left(\mathrm{CDCl}_{3}\right): 0.95(t, J$ $=7.4,3 \mathrm{H}), 1.44(\mathrm{~m}, 2 \mathrm{H}), 1.68(\mathrm{~m}, 2 \mathrm{H}), 2.44(\mathrm{~s}, 3 \mathrm{H}), 3.60(t, J=$ $7.1 \mathrm{~Hz}, 2 \mathrm{H}), 7.10(t, J=5.2 \mathrm{~Hz}, 1 \mathrm{H}), 7.78(t, J=7.0 \mathrm{~Hz}, 1 \mathrm{H})$, $7.93(d, J=8.4 \mathrm{~Hz}, 1 \mathrm{H}), 8.28(d, J=4.8 \mathrm{~Hz}, 1 \mathrm{H}), 9.82(s, 1 \mathrm{H})$; ${ }^{13} \mathrm{C}$ NMR $\left(\mathrm{CDCl}_{3}\right): 13.7\left(\mathrm{CH}_{3}\right), 14.5\left(\mathrm{CH}_{3}\right), 19.9\left(\mathrm{CH}_{2}\right), 32.0$ $\left(\mathrm{CH}_{2}\right), 46.4\left(\mathrm{CH}_{2}\right), 106.6(\mathrm{C}), 114.0(\mathrm{CH}), 119.8(\mathrm{CH}), 138.8$ $(\mathrm{CH}), 145.8(\mathrm{CH}), 152.8(\mathrm{C}), 153.0(\mathrm{C}), 154.3(\mathrm{C}), 182.0(\mathrm{CH})$; MS (EI) $m / z 258\left(M^{+}, 26 \%\right), 215$ (67), 187 (59), 134 (32), 93 (47), 78 (76), 51 (24), 32 (100); HRMS $m / z$ (ESI) calculated for $\left[\mathrm{C}_{14} \mathrm{H}_{18} \mathrm{~N}_{4} \mathrm{O}+\mathrm{H}\right]^{+}:$259.1553; found $259.1546\left[(M+\mathrm{H})^{+}\right]$.

\section{Refinement}

Crystal data, data collection and structure refinement details are summarized in Table $2 . \mathrm{H}$ atoms were placed in calculated positions $(\mathrm{C}-\mathrm{H}=0.95-0.99 \AA)$ and included as riding with isotropic displacement parameters set at 1.2-1.5 times the $U_{\text {eq }}$ value of the parent atom. $\mathrm{H}$ atoms belonging to $\mathrm{NH}$ groups were located in difference density maps and were freely refined.

\section{Acknowledgements}

The authors are grateful for financial support from the Universidad de los Andes and the Colombian Institute for Science and Research (COLCIENCIAS).

\section{References}

Blessing, R. H. (1995). Acta Cryst. A51, 33-38.

Bruker (2008). SADABS. Bruker AXS Inc., Madison, Wisconsin, USA.
Table 2

Experimental details.

\begin{tabular}{|c|c|}
\hline \multicolumn{2}{|l|}{ Crystal data } \\
\hline Chemical formula & $\mathrm{C}_{14} \mathrm{H}_{18} \mathrm{~N}_{4} \mathrm{O}$ \\
\hline$M_{\mathrm{r}}$ & 258.32 \\
\hline Crystal system, space group & Monoclinic, $P 2_{1} / c$ \\
\hline Temperature $(\mathrm{K})$ & 100 \\
\hline$a, b, c(\AA)$ & $\begin{array}{l}9.2854(2), 7.59144(18), \\
19.4452(5)\end{array}$ \\
\hline$\beta\left(^{\circ}\right)$ & $102.818(3)$ \\
\hline$V\left(\AA^{3}\right)$ & $1336.52(6)$ \\
\hline$Z$ & 4 \\
\hline Radiation type & Mo $K \alpha$ \\
\hline$\mu\left(\mathrm{mm}^{-1}\right)$ & 0.09 \\
\hline Crystal size (mm) & $0.10 \times 0.10 \times 0.05$ \\
\hline
\end{tabular}

Data collection

Diffractometer

Absorption correction

$T_{\min }, T_{\max }$

No. of measured, independent and observed $[I>2 \sigma(I)]$ reflections

$R_{\text {int }}$

$(\sin \theta / \lambda)_{\max }\left(\AA^{-1}\right)$

Rigaku MicroMax-007HF

Multi-scan [SADABS (Bruker, 2008) and Blessing (1995)]

$0.766,0.996$

$14709,6368,5580$

0.016

0.848

Refinement

$R\left[F^{2}>2 \sigma\left(F^{2}\right)\right], w R\left(F^{2}\right), S$

No. of reflections

No. of parameters

$\mathrm{H}$-atom treatment

$\Delta \rho_{\max }, \Delta \rho_{\min }\left(\mathrm{e} \AA^{-3}\right)$

$0.037,0.110,1.05$
6368
178
$\mathrm{H}$ atoms treated by a mixture of
$\quad$ independent and constrained
$\quad$ refinement
$0.51,-0.23$

Computer programs: APEX2 andSAINT (Bruker, 2011), SIR2011 (Burla et al., 2012), SHELXL2014 (Sheldrick, 2015) and Mercury (Macrae et al., 2008).

Bruker (2011). APEX2 and SAINT. Bruker AXS Inc., Madison, Wisconsin, USA.

Budzisz, E., Miernicka, M., Lorenz, I.-P., Mayer, P., Krajewska, U. \& Rozalski, M. (2009). Polyhedron, 28, 637-645.

Burla, M. C., Caliandro, R., Camalli, M., Carrozzini, B., Cascarano, G. L., Giacovazzo, C., Mallamo, M., Mazzone, A., Polidori, G. \& Spagna, R. (2012). J. Appl. Cryst. 45, 357-361.

Groom, C. R., Bruno, I. J., Lightfoot, M. P. \& Ward, S. C. (2016). Acta Cryst. B72, 171-179.

Macrae, C. F., Bruno, I. J., Chisholm, J. A., Edgington, P. R., McCabe, P., Pidcock, E., Rodriguez-Monge, L., Taylor, R., van de Streek, J. \& Wood, P. A. (2008). J. Appl. Cryst. 41, 466-470.

Orrego-Hernández, J., Cobo, J. \& Portilla, J. (2015a). Eur. J. Org. Chem. pp. 5064-5069.

Orrego Hernández, J., Portilla, J., Cobo, J. \& Glidewell, C. (2015b). Acta Cryst. C71, 363-368.

Peng, X.-M., Cai, G.-X. \& Zhou, C.-H. (2013). Curr. Top. Med. Chem. 13, 1963-2010.

Sakya, S. M., Lundy DeMello, K. M., Minich, M. L., Rast, B., Shavnya, A., Rafka, R. J., Koss, D. A., Cheng, H., Li, J., Jaynes, B. H., Ziegler, C. B., Mann, D. W., Petras, C. F., Seibel, S. B., Silvia, A. M., George, D. M., Lund, L. A., Denis, S. S., Hickman, A., Haven, M. L. \& Lynch, M. P. (2006). Bioorg. Med. Chem. Lett. 16, 288-292.

Sheldrick, G. M. (2015). Acta Cryst. C71, 3-8.

Yuan, J.-G., Wu, H.-X., Lu, M.-L., Song, G.-P. \& Xu, H.-H. (2013). J. Agric. Food Chem. 61, 4236-4241.

Zhang, Z., Ojo, K. K., Vidadala, R., Huang, W., Geiger, J. A., Scheele, S., Choi, R., Reid, M. C., Keyloun, K. R., Rivas, K., Siddaramaiah, L. K., Comess, K. M., Robinson, K. P., Merta, P. J., Kifle, L., Hol, W. G. J., Parsons, M., Merritt, E. A., Maly, D. J., Verlinde, C. L. M. J., Van Voorhis, W. C. \& Fan, E. (2014). ACS Med. Chem. Lett. 5, 4044. 


\section{supporting information}

Acta Cryst. (2016). E72, 1672-1674 [https://doi.org/10.1107/S2056989016017187]

Crystal structure of 5-butylamino-3-methyl-1-(pyridin-2-yl)-1 H-pyrazole-4-

carbaldehyde obtained from a microwave-assisted reaction using caesium

carbonate as catalyst

Mario A. Macías, Jessica Orrego-Hernández and Jaime Portilla

Computing details

Data collection: APEX2 (Bruker, 2011); cell refinement: APEX2 (Bruker, 2011); data reduction: SAINT (Bruker, 2011); program(s) used to solve structure: SIR2011 (Burla et al., 2012); program(s) used to refine structure: SHELXL2014 (Sheldrick, 2015); molecular graphics: Mercury (Macrae et al., 2008); software used to prepare material for publication: SHELXL2014 (Sheldrick, 2015).

5-Butylamino-3-methyl-1-(pyridin-2-yl)-1H-pyrazole-4-carbaldehyde

Crystal data

$\mathrm{C}_{14} \mathrm{H}_{18} \mathrm{~N}_{4} \mathrm{O}$

$M_{r}=258.32$

Monoclinic, $P 2{ }_{1} / c$

$a=9.2854(2) \AA$

$b=7.59144(18) \AA$

$c=19.4452(5) \AA$

$\beta=102.818(3)^{\circ}$

$V=1336.52(6) \AA^{3}$

$Z=4$

Data collection

Rigaku MicroMax-007HF diffractometer

Radiation source: Microfocus rotating anode Xray tube, Rigaku MicroMax-007HF

Confocal Max Flux optic monochromator Detector resolution: 512 pixels $\mathrm{mm}^{-1}$

Fullsphere data collection, phi and $\omega$ scans Absorption correction: multi-scan

[SADABS (Bruker, 2008) and Blessing (1995)]

\section{Refinement}

Refinement on $F^{2}$

Least-squares matrix: full

$R\left[F^{2}>2 \sigma\left(F^{2}\right)\right]=0.037$

$w R\left(F^{2}\right)=0.110$

$S=1.05$

6368 reflections

178 parameters
$F(000)=552$

$D_{\mathrm{x}}=1.284 \mathrm{Mg} \mathrm{m}^{-3}$

Mo $K \alpha$ radiation, $\lambda=0.71073 \AA$

Cell parameters from 5580 reflections

$\theta=2.2-37.1^{\circ}$

$\mu=0.09 \mathrm{~mm}^{-1}$

$T=100 \mathrm{~K}$

Block, yellow

$0.10 \times 0.10 \times 0.05 \mathrm{~mm}$

$T_{\min }=0.766, T_{\max }=0.996$

14709 measured reflections

6368 independent reflections

5580 reflections with $I>2 \sigma(I)$

$R_{\text {int }}=0.016$

$\theta_{\max }=37.1^{\circ}, \theta_{\min }=2.2^{\circ}$

$h=-15 \rightarrow 11$

$k=-12 \rightarrow 9$

$l=-26 \rightarrow 32$

0 restraints

Primary atom site location: structure-invariant direct methods

Secondary atom site location: difference Fourier map

Hydrogen site location: mixed 
$\mathrm{H}$ atoms treated by a mixture of independent and constrained refinement

$w=1 /\left[\sigma^{2}\left(F_{\mathrm{o}}^{2}\right)+(0.0645 P)^{2}+0.1608 P\right]$ where $P=\left(F_{\mathrm{o}}^{2}+2 F_{\mathrm{c}}^{2}\right) / 3$

$$
\begin{aligned}
& (\Delta / \sigma)_{\max }=0.002 \\
& \Delta \rho_{\max }=0.51 \mathrm{e} \AA^{-3} \\
& \Delta \rho_{\min }=-0.23 \mathrm{e} \AA^{-3}
\end{aligned}
$$

\section{Special details}

Experimental. It should be noted that the esd's of the cell dimensions are probably too low; they should be multiplied by a factor of 2 to 10

Geometry. All esds (except the esd in the dihedral angle between two 1.s. planes) are estimated using the full covariance matrix. The cell esds are taken into account individually in the estimation of esds in distances, angles and torsion angles; correlations between esds in cell parameters are only used when they are defined by crystal symmetry. An approximate

\begin{tabular}{|c|c|c|c|c|}
\hline & $x$ & $y$ & $z$ & $U_{\text {iso }} * / U_{\text {eq }}$ \\
\hline N1 & $0.36539(5)$ & $0.84047(6)$ & $1.01082(2)$ & $0.01366(8)$ \\
\hline H1 & $0.4865(12)$ & $0.6805(13)$ & $0.9303(5)$ & $0.026(2)^{*}$ \\
\hline N2 & $0.33841(5)$ & $0.91944(6)$ & $1.07161(2)$ & $0.01495(9)$ \\
\hline $\mathrm{C} 4$ & $0.56919(6)$ & $0.79751(7)$ & $1.09414(3)$ & $0.01398(9)$ \\
\hline $\mathrm{C} 3$ & $0.45948(6)$ & $0.89383(7)$ & $1.12030(3)$ & $0.01481(9)$ \\
\hline N5 & $0.55256(5)$ & 0.68697 (7) & $0.97014(3)$ & $0.01577(9)$ \\
\hline $\mathrm{C} 5$ & $0.50458(6)$ & $0.76672(7)$ & $1.02231(3)$ & $0.01291(9)$ \\
\hline $\mathrm{C} 51$ & $0.70133(6)$ & $0.62013(7)$ & $0.97538(3)$ & $0.01519(9)$ \\
\hline H51A & 0.7209 & 0.5222 & 1.0098 & $0.018 *$ \\
\hline H51B & 0.7741 & 0.7147 & 0.9921 & $0.018 *$ \\
\hline C41 & $0.70862(6)$ & $0.73665(8)$ & $1.13548(3)$ & $0.01890(11)$ \\
\hline H41 & 0.7729 & 0.6763 & 1.1117 & $0.023 *$ \\
\hline $\mathrm{O} 41$ & $0.74985(6)$ & $0.75754(8)$ & $1.19936(3)$ & $0.02881(12)$ \\
\hline C31 & $0.47057(7)$ & $0.96306(9)$ & $1.19315(3)$ & $0.02071(11)$ \\
\hline H31A & 0.5549 & 1.0435 & 1.2054 & $0.031^{*}$ \\
\hline H31B & 0.4843 & 0.8647 & 1.2266 & $0.031 *$ \\
\hline $\mathrm{H} 31 \mathrm{C}$ & 0.3797 & 1.0264 & 1.1952 & $0.031^{*}$ \\
\hline $\mathrm{C} 16$ & $0.16351(7)$ & $0.74104(9)$ & $0.83692(3)$ & $0.02033(11)$ \\
\hline H16 & 0.1776 & 0.6732 & 0.7979 & $0.024 *$ \\
\hline $\mathrm{C} 15$ & $0.03122(7)$ & $0.83021(9)$ & $0.83065(3)$ & $0.02048(11)$ \\
\hline H15 & -0.0437 & 0.8234 & 0.7886 & $0.025^{*}$ \\
\hline $\mathrm{C} 14$ & $0.01147(6)$ & $0.93004(8)$ & $0.88782(3)$ & $0.01946(11)$ \\
\hline H14 & -0.0779 & 0.9931 & 0.8853 & $0.023 *$ \\
\hline $\mathrm{C} 13$ & $0.12286(6)$ & $0.93706(8)$ & $0.94845(3)$ & $0.01672(10)$ \\
\hline H13 & 0.1119 & 1.0050 & 0.9880 & $0.020 *$ \\
\hline $\mathrm{C} 12$ & $0.25184(6)$ & $0.84098(7)$ & $0.94950(3)$ & $0.01387(9)$ \\
\hline N11 & $0.27354(6)$ & $0.74497(7)$ & $0.89524(3)$ & $0.01754(9)$ \\
\hline C52 & $0.71751(6)$ & $0.55527(8)$ & $0.90348(3)$ & $0.01585(10)$ \\
\hline H52A & 0.7075 & 0.6566 & 0.8707 & $0.019^{*}$ \\
\hline H52B & 0.6366 & 0.4715 & 0.8847 & $0.019^{*}$ \\
\hline C53 & 0.86547 (6) & $0.46467(8)$ & 0.90607 (3) & $0.01637(10)$ \\
\hline H53A & 0.9465 & 0.5504 & 0.9214 & $0.020 *$ \\
\hline H53B & 0.8789 & 0.3679 & 0.9410 & $0.020^{*}$ \\
\hline
\end{tabular}
(isotropic) treatment of cell esds is used for estimating esds involving 1.s. planes.

Fractional atomic coordinates and isotropic or equivalent isotropic displacement parameters $\left(\AA^{2}\right)$ 


\begin{tabular}{lllll} 
C54 & $0.87324(7)$ & $0.39047(9)$ & $0.83406(3)$ & $0.01989(11)$ \\
H54A & 0.9708 & 0.3383 & 0.8367 & $0.030^{*}$ \\
H54B & 0.8568 & 0.4855 & 0.7991 & $0.030^{*}$ \\
H54C & 0.7971 & 0.3000 & 0.8202 & $0.030^{*}$ \\
\hline
\end{tabular}

Atomic displacement parameters $\left(\AA^{2}\right)$

\begin{tabular}{lllllll}
\hline & $U^{11}$ & $U^{22}$ & $U^{33}$ & $U^{12}$ & $U^{13}$ & $U^{23}$ \\
\hline $\mathrm{N} 1$ & $0.01143(17)$ & $0.01598(19)$ & $0.01227(17)$ & $0.00075(14)$ & $-0.00017(14)$ & $0.00018(14)$ \\
$\mathrm{N} 2$ & $0.01383(19)$ & $0.01611(19)$ & $0.01371(18)$ & $0.00040(14)$ & $0.00052(14)$ & $-0.00137(14)$ \\
$\mathrm{C} 4$ & $0.01133(19)$ & $0.0156(2)$ & $0.0135(2)$ & $-0.00096(15)$ & $-0.00037(16)$ & $0.00017(16)$ \\
$\mathrm{C} 3$ & $0.0136(2)$ & $0.0155(2)$ & $0.0140(2)$ & $-0.00133(16)$ & $0.00010(16)$ & $-0.00071(16)$ \\
$\mathrm{N} 5$ & $0.01264(18)$ & $0.0202(2)$ & $0.01381(18)$ & $0.00099(15)$ & $0.00157(14)$ & $-0.00068(15)$ \\
C5 & $0.01084(19)$ & $0.0134(2)$ & $0.01358(19)$ & $-0.00073(15)$ & $0.00066(15)$ & $0.00134(15)$ \\
C51 & $0.0126(2)$ & $0.0166(2)$ & $0.0161(2)$ & $-0.00029(16)$ & $0.00243(16)$ & $-0.00001(16)$ \\
C41 & $0.0137(2)$ & $0.0238(3)$ & $0.0168(2)$ & $0.00154(18)$ & $-0.00179(18)$ & $-0.00114(19)$ \\
O41 & $0.0213(2)$ & $0.0437(3)$ & $0.0168(2)$ & $0.0072(2)$ & $-0.00562(17)$ & $-0.00410(19)$ \\
C31 & $0.0202(2)$ & $0.0248(3)$ & $0.0153(2)$ & $0.0004(2)$ & $0.00020(19)$ & $-0.00476(19)$ \\
C16 & $0.0190(2)$ & $0.0280(3)$ & $0.0119(2)$ & $-0.0014(2)$ & $-0.00112(18)$ & $0.00153(19)$ \\
C15 & $0.0163(2)$ & $0.0270(3)$ & $0.0152(2)$ & $-0.0032(2)$ & $-0.00273(18)$ & $0.00527(19)$ \\
C14 & $0.0135(2)$ & $0.0217(3)$ & $0.0204(2)$ & $-0.00041(18)$ & $-0.00236(18)$ & $0.00482(19)$ \\
C13 & $0.0127(2)$ & $0.0171(2)$ & $0.0183(2)$ & $0.00055(16)$ & $-0.00094(17)$ & $0.00179(17)$ \\
C12 & $0.01192(19)$ & $0.0151(2)$ & $0.0130(2)$ & $-0.00119(15)$ & $-0.00053(15)$ & $0.00273(15)$ \\
N11 & $0.0159(2)$ & $0.0230(2)$ & $0.01223(18)$ & $0.00039(16)$ & $-0.00005(15)$ & $0.00079(15)$ \\
C52 & $0.0141(2)$ & $0.0184(2)$ & $0.0148(2)$ & $0.00027(17)$ & $0.00264(16)$ & $0.00057(17)$ \\
C53 & $0.0143(2)$ & $0.0196(2)$ & $0.0152(2)$ & $0.00035(17)$ & $0.00317(17)$ & $0.00079(17)$ \\
C54 & $0.0181(2)$ & $0.0246(3)$ & $0.0172(2)$ & $0.0018(2)$ & $0.00455(19)$ & $-0.00125(19)$ \\
& & & & & &
\end{tabular}

Geometric parameters $\left(\AA,{ }^{\circ}\right)$

\begin{tabular}{llll}
\hline $\mathrm{N} 1-\mathrm{C} 5$ & $1.3804(7)$ & $\mathrm{C} 16-\mathrm{N} 11$ & $1.3478(7)$ \\
$\mathrm{N} 1-\mathrm{N} 2$ & $1.3968(7)$ & $\mathrm{C} 16-\mathrm{C} 15$ & $1.3841(9)$ \\
$\mathrm{N} 1-\mathrm{C} 12$ & $1.4056(7)$ & $\mathrm{C} 16-\mathrm{H} 16$ & 0.9500 \\
$\mathrm{~N} 2-\mathrm{C} 3$ & $1.3137(7)$ & $\mathrm{C} 15-\mathrm{C} 14$ & $1.3909(9)$ \\
$\mathrm{C} 4-\mathrm{C} 5$ & $1.4119(7)$ & $\mathrm{C} 15-\mathrm{H} 15$ & 0.9500 \\
$\mathrm{C} 4-\mathrm{C} 3$ & $1.4357(8)$ & $\mathrm{C} 14-\mathrm{C} 13$ & $1.3862(8)$ \\
$\mathrm{C} 4-\mathrm{C} 41$ & $1.4403(8)$ & $\mathrm{C} 14-\mathrm{H} 14$ & 0.9500 \\
$\mathrm{C} 3-\mathrm{C} 31$ & $1.4928(8)$ & $\mathrm{C} 13-\mathrm{C} 12$ & $1.3986(8)$ \\
$\mathrm{N} 5-\mathrm{C} 5$ & $1.3396(7)$ & $\mathrm{C} 13-\mathrm{H} 13$ & 0.9500 \\
$\mathrm{~N} 5-\mathrm{C} 51$ & $1.4539(7)$ & $\mathrm{C} 12-\mathrm{N} 11$ & $1.3340(8)$ \\
$\mathrm{N} 5-\mathrm{H} 1$ & $0.876(10)$ & $\mathrm{C} 52-\mathrm{C} 53$ & $1.5272(8)$ \\
$\mathrm{C} 51-\mathrm{C} 52$ & $1.5208(8)$ & $\mathrm{C} 52-\mathrm{H} 52 \mathrm{~A}$ & 0.9900 \\
$\mathrm{C} 51-\mathrm{H} 51 \mathrm{~A}$ & 0.9900 & $\mathrm{C} 52-\mathrm{H} 52 \mathrm{~B}$ & 0.9900 \\
$\mathrm{C} 51-\mathrm{H} 51 \mathrm{~B}$ & 0.9900 & $\mathrm{C} 53-\mathrm{C} 54$ & $1.5258(8)$ \\
$\mathrm{C} 41-\mathrm{O} 41$ & $1.2261(7)$ & $\mathrm{C} 53-\mathrm{H} 53 \mathrm{~A}$ & 0.9900 \\
$\mathrm{C} 41-\mathrm{H} 41$ & 0.9500 & $\mathrm{C} 53-\mathrm{H} 53 \mathrm{~B}$ & 0.9900 \\
$\mathrm{C} 31-\mathrm{H} 31 \mathrm{~A}$ & 0.9800 & $\mathrm{C} 54-\mathrm{H} 54 \mathrm{~A}$ & 0.9800 \\
$\mathrm{C} 31-\mathrm{H} 31 \mathrm{~B}$ & 0.9800 & $\mathrm{C} 54-\mathrm{H} 54 \mathrm{~B}$ & 0.9800
\end{tabular}




\begin{tabular}{|c|c|c|c|}
\hline $\mathrm{C} 31-\mathrm{H} 31 \mathrm{C}$ & 0.9800 & $\mathrm{C} 54-\mathrm{H} 54 \mathrm{C}$ & 0.9800 \\
\hline $\mathrm{C} 5-\mathrm{N} 1-\mathrm{N} 2$ & $111.99(4)$ & $\mathrm{C} 15-\mathrm{C} 16-\mathrm{H} 16$ & 118.1 \\
\hline $\mathrm{C} 5-\mathrm{N} 1-\mathrm{C} 12$ & $129.61(5)$ & $\mathrm{C} 16-\mathrm{C} 15-\mathrm{C} 14$ & $117.90(5)$ \\
\hline $\mathrm{N} 2-\mathrm{N} 1-\mathrm{C} 12$ & $118.37(4)$ & $\mathrm{C} 16-\mathrm{C} 15-\mathrm{H} 15$ & 121.1 \\
\hline $\mathrm{C} 3-\mathrm{N} 2-\mathrm{N} 1$ & $105.12(4)$ & $\mathrm{C} 14-\mathrm{C} 15-\mathrm{H} 15$ & 121.1 \\
\hline $\mathrm{C} 5-\mathrm{C} 4-\mathrm{C} 3$ & $104.82(5)$ & $\mathrm{C} 13-\mathrm{C} 14-\mathrm{C} 15$ & $119.67(6)$ \\
\hline $\mathrm{C} 5-\mathrm{C} 4-\mathrm{C} 41$ & $129.14(5)$ & $\mathrm{C} 13-\mathrm{C} 14-\mathrm{H} 14$ & 120.2 \\
\hline $\mathrm{C} 3-\mathrm{C} 4-\mathrm{C} 41$ & $125.89(5)$ & $\mathrm{C} 15-\mathrm{C} 14-\mathrm{H} 14$ & 120.2 \\
\hline $\mathrm{N} 2-\mathrm{C} 3-\mathrm{C} 4$ & $112.39(5)$ & $\mathrm{C} 14-\mathrm{C} 13-\mathrm{C} 12$ & $117.86(6)$ \\
\hline $\mathrm{N} 2-\mathrm{C} 3-\mathrm{C} 31$ & $119.97(5)$ & $\mathrm{C} 14-\mathrm{C} 13-\mathrm{H} 13$ & 121.1 \\
\hline $\mathrm{C} 4-\mathrm{C} 3-\mathrm{C} 31$ & $127.63(5)$ & $\mathrm{C} 12-\mathrm{C} 13-\mathrm{H} 13$ & 121.1 \\
\hline $\mathrm{C} 5-\mathrm{N} 5-\mathrm{C} 51$ & $125.11(5)$ & $\mathrm{N} 11-\mathrm{C} 12-\mathrm{C} 13$ & $123.54(5)$ \\
\hline $\mathrm{C} 5-\mathrm{N} 5-\mathrm{H} 1$ & $114.2(7)$ & $\mathrm{N} 11-\mathrm{C} 12-\mathrm{N} 1$ & $116.94(5)$ \\
\hline $\mathrm{C} 51-\mathrm{N} 5-\mathrm{H} 1$ & $120.7(7)$ & $\mathrm{C} 13-\mathrm{C} 12-\mathrm{N} 1$ & $119.51(5)$ \\
\hline $\mathrm{N} 5-\mathrm{C} 5-\mathrm{N} 1$ & $121.17(5)$ & $\mathrm{C} 12-\mathrm{N} 11-\mathrm{C} 16$ & $117.28(5)$ \\
\hline $\mathrm{N} 5-\mathrm{C} 5-\mathrm{C} 4$ & $133.17(5)$ & $\mathrm{C} 51-\mathrm{C} 52-\mathrm{C} 53$ & $112.78(5)$ \\
\hline $\mathrm{N} 1-\mathrm{C} 5-\mathrm{C} 4$ & $105.66(5)$ & $\mathrm{C} 51-\mathrm{C} 52-\mathrm{H} 52 \mathrm{~A}$ & 109.0 \\
\hline $\mathrm{N} 5-\mathrm{C} 51-\mathrm{C} 52$ & $109.55(4)$ & $\mathrm{C} 53-\mathrm{C} 52-\mathrm{H} 52 \mathrm{~A}$ & 109.0 \\
\hline N5-C51-H51A & 109.8 & $\mathrm{C} 51-\mathrm{C} 52-\mathrm{H} 52 \mathrm{~B}$ & 109.0 \\
\hline $\mathrm{C} 52-\mathrm{C} 51-\mathrm{H} 51 \mathrm{~A}$ & 109.8 & $\mathrm{C} 53-\mathrm{C} 52-\mathrm{H} 52 \mathrm{~B}$ & 109.0 \\
\hline N5-C51-H51B & 109.8 & $\mathrm{H} 52 \mathrm{~A}-\mathrm{C} 52-\mathrm{H} 52 \mathrm{~B}$ & 107.8 \\
\hline $\mathrm{C} 52-\mathrm{C} 51-\mathrm{H} 51 \mathrm{~B}$ & 109.8 & $\mathrm{C} 54-\mathrm{C} 53-\mathrm{C} 52$ & $111.19(5)$ \\
\hline $\mathrm{H} 51 \mathrm{~A}-\mathrm{C} 51-\mathrm{H} 51 \mathrm{~B}$ & 108.2 & $\mathrm{C} 54-\mathrm{C} 53-\mathrm{H} 53 \mathrm{~A}$ & 109.4 \\
\hline $\mathrm{O} 41-\mathrm{C} 41-\mathrm{C} 4$ & $124.33(6)$ & $\mathrm{C} 52-\mathrm{C} 53-\mathrm{H} 53 \mathrm{~A}$ & 109.4 \\
\hline $\mathrm{O} 41-\mathrm{C} 41-\mathrm{H} 41$ & 117.8 & C54-C53-H53B & 109.4 \\
\hline $\mathrm{C} 4-\mathrm{C} 41-\mathrm{H} 41$ & 117.8 & $\mathrm{C} 52-\mathrm{C} 53-\mathrm{H} 53 \mathrm{~B}$ & 109.4 \\
\hline $\mathrm{C} 3-\mathrm{C} 31-\mathrm{H} 31 \mathrm{~A}$ & 109.5 & $\mathrm{H} 53 \mathrm{~A}-\mathrm{C} 53-\mathrm{H} 53 \mathrm{~B}$ & 108.0 \\
\hline $\mathrm{C} 3-\mathrm{C} 31-\mathrm{H} 31 \mathrm{~B}$ & 109.5 & $\mathrm{C} 53-\mathrm{C} 54-\mathrm{H} 54 \mathrm{~A}$ & 109.5 \\
\hline $\mathrm{H} 31 \mathrm{~A}-\mathrm{C} 31-\mathrm{H} 31 \mathrm{~B}$ & 109.5 & $\mathrm{C} 53-\mathrm{C} 54-\mathrm{H} 54 \mathrm{~B}$ & 109.5 \\
\hline $\mathrm{C} 3-\mathrm{C} 31-\mathrm{H} 31 \mathrm{C}$ & 109.5 & $\mathrm{H} 54 \mathrm{~A}-\mathrm{C} 54-\mathrm{H} 54 \mathrm{~B}$ & 109.5 \\
\hline $\mathrm{H} 31 \mathrm{~A}-\mathrm{C} 31-\mathrm{H} 31 \mathrm{C}$ & 109.5 & $\mathrm{C} 53-\mathrm{C} 54-\mathrm{H} 54 \mathrm{C}$ & 109.5 \\
\hline $\mathrm{H} 31 \mathrm{~B}-\mathrm{C} 31-\mathrm{H} 31 \mathrm{C}$ & 109.5 & $\mathrm{H} 54 \mathrm{~A}-\mathrm{C} 54-\mathrm{H} 54 \mathrm{C}$ & 109.5 \\
\hline $\mathrm{N} 11-\mathrm{C} 16-\mathrm{C} 15$ & $123.75(6)$ & $\mathrm{H} 54 \mathrm{~B}-\mathrm{C} 54-\mathrm{H} 54 \mathrm{C}$ & 109.5 \\
\hline $\mathrm{N} 11-\mathrm{C} 16-\mathrm{H} 16$ & 118.1 & & \\
\hline $\mathrm{C} 5-\mathrm{N} 1-\mathrm{N} 2-\mathrm{C} 3$ & $-0.35(6)$ & $\mathrm{C} 5-\mathrm{N} 5-\mathrm{C} 51-\mathrm{C} 52$ & $-174.43(5)$ \\
\hline $\mathrm{C} 12-\mathrm{N} 1-\mathrm{N} 2-\mathrm{C} 3$ & $177.72(5)$ & $\mathrm{C} 5-\mathrm{C} 4-\mathrm{C} 41-\mathrm{O} 41$ & $-173.30(7)$ \\
\hline $\mathrm{N} 1-\mathrm{N} 2-\mathrm{C} 3-\mathrm{C} 4$ & $-0.34(6)$ & $\mathrm{C} 3-\mathrm{C} 4-\mathrm{C} 41-\mathrm{O} 41$ & $1.38(10)$ \\
\hline $\mathrm{N} 1-\mathrm{N} 2-\mathrm{C} 3-\mathrm{C} 31$ & $179.52(5)$ & $\mathrm{N} 11-\mathrm{C} 16-\mathrm{C} 15-\mathrm{C} 14$ & $0.30(10)$ \\
\hline $\mathrm{C} 5-\mathrm{C} 4-\mathrm{C} 3-\mathrm{N} 2$ & $0.88(6)$ & $\mathrm{C} 16-\mathrm{C} 15-\mathrm{C} 14-\mathrm{C} 13$ & $-0.09(9)$ \\
\hline $\mathrm{C} 41-\mathrm{C} 4-\mathrm{C} 3-\mathrm{N} 2$ & $-174.85(5)$ & $\mathrm{C} 15-\mathrm{C} 14-\mathrm{C} 13-\mathrm{C} 12$ & $-0.37(9)$ \\
\hline $\mathrm{C} 5-\mathrm{C} 4-\mathrm{C} 3-\mathrm{C} 31$ & $-178.97(6)$ & $\mathrm{C} 14-\mathrm{C} 13-\mathrm{C} 12-\mathrm{N} 11$ & $0.69(9)$ \\
\hline $\mathrm{C} 41-\mathrm{C} 4-\mathrm{C} 3-\mathrm{C} 31$ & $5.29(10)$ & $\mathrm{C} 14-\mathrm{C} 13-\mathrm{C} 12-\mathrm{N} 1$ & $-178.05(5)$ \\
\hline $\mathrm{C} 51-\mathrm{N} 5-\mathrm{C} 5-\mathrm{N} 1$ & $174.02(5)$ & $\mathrm{C} 5-\mathrm{N} 1-\mathrm{C} 12-\mathrm{N} 11$ & $6.28(8)$ \\
\hline $\mathrm{C} 51-\mathrm{N} 5-\mathrm{C} 5-\mathrm{C} 4$ & $-5.74(10)$ & $\mathrm{N} 2-\mathrm{N} 1-\mathrm{C} 12-\mathrm{N} 11$ & $-171.40(5)$ \\
\hline $\mathrm{N} 2-\mathrm{N} 1-\mathrm{C} 5-\mathrm{N} 5$ & $-178.93(5)$ & $\mathrm{C} 5-\mathrm{N} 1-\mathrm{C} 12-\mathrm{C} 13$ & $-174.90(5)$ \\
\hline $\mathrm{C} 12-\mathrm{N} 1-\mathrm{C} 5-\mathrm{N} 5$ & $3.28(9)$ & $\mathrm{N} 2-\mathrm{N} 1-\mathrm{C} 12-\mathrm{C} 13$ & $7.42(7)$ \\
\hline
\end{tabular}




$\begin{array}{llll}\mathrm{N} 2-\mathrm{N} 1-\mathrm{C} 5-\mathrm{C} 4 & 0.89(6) & \mathrm{C} 13-\mathrm{C} 12-\mathrm{N} 11-\mathrm{C} 16 & -0.49(9) \\ \mathrm{C} 12-\mathrm{N} 1-\mathrm{C} 5-\mathrm{C} 4 & -176.91(5) & \mathrm{N} 1-\mathrm{C} 12-\mathrm{N} 11-\mathrm{C} 16 & 178.28(5) \\ \mathrm{C} 3-\mathrm{C} 4-\mathrm{C} 5-\mathrm{N} 5 & 178.77(6) & \mathrm{C} 15-\mathrm{C} 16-\mathrm{N} 11-\mathrm{C} 12 & -0.02(9) \\ \mathrm{C} 41-\mathrm{C} 4-\mathrm{C} 5-\mathrm{N} 5 & -5.69(10) & \mathrm{N} 5-\mathrm{C} 51-\mathrm{C} 52-\mathrm{C} 53 & -173.79(5) \\ \mathrm{C} 3-\mathrm{C} 4-\mathrm{C} 5-\mathrm{N} 1 & -1.02(6) & \mathrm{C} 51-\mathrm{C} 52-\mathrm{C} 53-\mathrm{C} 54 & 176.10(5) \\ \mathrm{C} 41-\mathrm{C} 4-\mathrm{C} 5-\mathrm{N} 1 & 174.53(6) & & \end{array}$

Hydrogen-bond geometry $\left(A,{ }^{\circ}\right)$

$\mathrm{Cg} 1$ abd $\mathrm{Cg} 2$ are the centroids of the $\mathrm{C} 3-\mathrm{C} 5 / \mathrm{N} 1 / \mathrm{N} 2$ and $\mathrm{N} 11 / \mathrm{C} 12-\mathrm{C} 16$ rings, respectively.

\begin{tabular}{lllll}
\hline$D-\mathrm{H} \cdots A$ & $D-\mathrm{H}$ & $\mathrm{H} \cdots A$ & $D \cdots A$ & $D-\mathrm{H} \cdots A$ \\
\hline $\mathrm{N} 5-\mathrm{H} 1 \cdots \mathrm{N} 11$ & $0.876(10)$ & $2.004(11)$ & $2.7117(7)$ & $137.0(9)$ \\
$\mathrm{C} 15-\mathrm{H} 15 \cdots \mathrm{O} 41^{\mathrm{i}}$ & 0.95 & 2.36 & $3.2906(8)$ & 165 \\
$\mathrm{C} 52-\mathrm{H} 52 B \cdots C g 1^{\mathrm{ii}}$ & 0.99 & 2.77 & $3.5141(6)$ & 132 \\
$\mathrm{C} 53-\mathrm{H} 53 A \cdots C g 2^{\mathrm{iii}}$ & 0.99 & 2.98 & $3.8761(6)$ & 152
\end{tabular}

Symmetry codes: (i) $x-1,-y+3 / 2, z-1 / 2$; (ii) $-x+1,-y+1,-z+2$; (iii) $x+1, y, z$. 